PUPT-1364

UBCTP 92-033

Revised Version

February, 1993

\title{
THE SPECTRUM OF TOPOLOGICALLY MASSIVE QUANTUM ELECTRODYNAMICS
}

\author{
Mikhail I. Dobroliubov $(a), *$, David Eliezer ${ }^{(b)}$, Ian I. Kogan ${ }^{(c), * *}$, \\ Gordon W. Semenoff ${ }^{(a)}$ and Richard J. Szabo ${ }^{(a)}$ \\ ${ }^{(a)}$ Department of Physics, University of British Columbia \\ Vancouver, British Columbia, V6T $1 Z 1$ Canada \\ (b) Department of Physics, L-412 \\ Lawrence Livermore National Laboratory \\ P.O. Box 808, Livermore, California, 94551-9900 U.S.A. \\ (c) Joseph Henry Laboratory \\ Department of Physics, Princeton University \\ Princeton, New Jersey, 08544 U.S.A.
}

\begin{abstract}
We discuss the possibility for the spectrum of topologically massive quantum electrodynamics with spinor matter fields to contain unexpected and unusual stable particle excitations for certain values of the topological photon mass. The new field theoretical phenomena arising from this novel spectral structure are briefly discussed.
\end{abstract}

PACS Numbers: 11.10.St, 12.20.Ds, 03.65.Ge

* Permanent Address: Institute for Nuclear Research, Academy of Sciences of Russia, Moscow, 117312 Russia.

** Permanent Address: Institute for Theoretical and Experimental Physics, B. Cheremushkinskaya ul. 25, Moscow, 117259 Russia. 
One of the most interesting features of quantum electrodynamics in $(2+1)$ dimensions is the possibility that the photon can have a gauge invariant topological mass term [1]. The Lagrangian contains both a Maxwell term and a parity and time-reversal violating Chern-Simons kinetic term for the gauge field as well as minimal coupling to spinor matter:

$$
\mathcal{L}=-\frac{1}{4 e^{2}} F_{\mu \nu} F^{\mu \nu}+\frac{k}{8 \pi} \epsilon^{\mu \nu \lambda} A_{\mu} \partial_{\nu} A_{\lambda}+\bar{\psi}\left(i \gamma^{\mu} \mathcal{D}_{\mu}-m\right) \psi
$$

Here $A$ is an Abelian vector field, $F_{\mu \nu}=\partial_{\mu} A_{\nu}-\partial_{\nu} A_{\mu}$ is the field strength tensor, $\mathcal{D}_{\mu}=$ $\partial_{\mu}-i A_{\mu}$ is the gauge covariant derivative, and $\psi$ is a two-component Dirac field.

This model effectively contains two dimensionful parameters, the topological photon mass $M=e^{2} k / 4 \pi$, which may be positive or negative depending on the sign of the statistics parameter $k$, and the electron mass $m$, which may also assume both positive and negative values, but we choose $m>0$ for certainty. The topological mass cuts off the long range Coulomb interaction which in the pure Maxwell theory would be logarithmic. There are two natural dimensionless parameters in this model, the ratios $\alpha / m$ and $\alpha / M=1 / k$, where $\alpha=e^{2} / 4 \pi$. For light fermions $(m<M)$ the perturbation series expansion parameter is $\alpha^{2} / m M \sim 1 / k$, while for heavy fermions $(m \gg M)$ it turns out to be $\alpha / m=(M / m)(1 / k) \ll 1 / k$. The standard perturbation series can therefore be used provided $1 / k \ll 1$.

Drawing from our experience with $\mathrm{QED}_{4}$, one would expect that for large $k$ the spectrum of the theory (1) is described well by perturbation theory. It contains the tree-level particles, the photon, electron and positron, and also, because of the attractive Coulomb interaction, metastable $e^{+} e^{-}$bound states, as expected from the analysis of ordinary $\mathrm{QED}_{3}$ $(k \rightarrow 0$ in $(1))$. In the following we shall show that in fact the qualitative properties of the spectrum are controlled not only by the statistics parameter $k$, but also by the parameter $\beta \equiv M / m=k e^{2} / 4 \pi m$. When the parameter $\beta$ is small, the photon is relatively light, the theory resembles pure $\mathrm{QED}_{3}$, and the spectrum contains electrons, positrons, photons and $e^{+} e^{-}$bound states.

Also, we shall see that when $\beta$ is increased to 1 , a new phenomenon takes place - the equal charge attraction which was originally discussed in Ref. [2]. In this case an attractive charge-current interaction and repulsive charge-charge and current-current interactions become of comparable strength and may lead to new $e^{-} e^{-}$and $e^{+} e^{+}$bound states [2-7]. When $M / m>1$, the $e^{+} e^{-}$bound states no longer form. Instead, for small enough $k \lesssim 1$ (the strong coupling regime), as well as the electron, positron and photon, the spectrum 
of the theory may contain scalar (if there are several fermion flavours in the theory) and vector $e^{-} e^{-}$and $e^{+} e^{+}$bound states. We speculate that for a special set of parameters, the vector $e^{-} e^{-}$and $e^{+} e^{+}$bound states and the photon have equal masses and form an $\mathrm{SU}(2)$ isospin triplet state, although at this point we have not yet determined whether the dynamics could exhibit a full global SU(2) symmetry.

The situation again changes at the two-fermion threshold $M / m=2$ where the photon becomes unstable to decay into $e^{+} e^{-}$pairs. Here we shall show that an unusual attraction between the electron or positron and photon as well as $e^{+} e^{-}$repulsion take place. There, we conjecture that, for a certain range of parameters, the stable particles are an $e^{-} \gamma$ bound state and an $e^{+} \gamma$ bound state, as well as an electron and positron and possible $e^{-} e^{-}$and $e^{+} e^{+}$bound states (and then if the masses of the bound states are light enough, the electron and positron could actually be unstable and decay into an $e^{-} \gamma$ or $e^{+} \gamma$ bound state and a photon). As the parameter $M / m$ becomes infinitely large, however, these unusual particle interactions only represent fractional spin-statistics transmutations of the composite particle systems, and the only stable particles which remain are the electron and positron.

The $e^{-} e^{-}$interaction in the theory (1) can be described in perturbation theory by considering the fermion-fermion scattering amplitude in the non-relativistic limit. Working in the tree-approximation, for different flavour fermions we symmetrize the two corresponding $t$ - and $u$-channel diagrams to get the total symmetric amplitude $A_{\mathrm{s}}$, while for identical fermions the Pauli exclusion principle forces us to antisymmetrize and get the total antisymmetric amplitude $A_{\mathrm{as}}$ :

$$
\begin{aligned}
A_{\mathrm{S}, \mathrm{as}}=-\frac{i}{2}[( & \left.\bar{u}\left(p_{1}^{\prime}\right) \gamma^{\mu} u\left(p_{1}\right)\right)\left(\bar{u}\left(p_{2}^{\prime}\right) \gamma^{\nu} u\left(p_{2}\right)\right) G_{\mu \nu}\left(p_{1}-p_{1}^{\prime}\right) \\
& \left. \pm\left(\bar{u}\left(p_{2}^{\prime}\right) \gamma^{\mu} u\left(p_{1}\right)\right)\left(\bar{u}\left(p_{1}^{\prime}\right) \gamma^{\nu} u\left(p_{2}\right)\right) G_{\mu \nu}\left(p_{1}-p_{2}^{\prime}\right)\right] .
\end{aligned}
$$

Here $p_{i}\left(p_{i}^{\prime}\right)$ are the momenta of the incoming (outgoing) particles, and

$$
u(p)=\frac{1}{\sqrt{2 m(E-m)}}\left(\begin{array}{c}
E+m \\
-i\left(p_{1}+i p_{2}\right)
\end{array}\right),
$$

where $p=\left(E, p_{1}, p_{2}\right), E^{2}=\vec{p}^{2}+m^{2}$, and $\bar{u}(p) u(p)=1$, are the on-shell positive energy Dirac spinors [6]. We also use the $(2+1)$ dimensional representation of the Dirac matrices in terms of Pauli spin matrices as $\gamma_{0}=\sigma^{3}, \gamma_{1}=i \sigma^{1}$, and $\gamma_{2}=i \sigma^{2}$, and

$$
G_{\mu \nu}(p)=-i e^{2}\left[\frac{p^{2} g_{\mu \nu}-p_{\mu} p_{\nu}}{p^{2}\left(p^{2}-M^{2}\right)}+\frac{i M \epsilon_{\mu \nu \lambda} p^{\lambda}}{p^{2}\left(p^{2}-M^{2}\right)}\right]
$$


is the free photon propagator in the Landau gauge [1].

The imaginary parts of the $S$-matrix elements (2) describe the lowest order long-ranged Aharonov-Bohm flux tube interaction of the electrons, which vanishes in the short-ranged regime and in the long distance limit just shifts the canonical angular momentum of the charged particles as $\ell \rightarrow \ell-\frac{1}{k}$. The real parts of these amplitudes, which describe the short-ranged interactions between the electrons, in the center of mass frame and in the non-relativistic limit are

$$
\operatorname{Re}\left(A_{\mathrm{s}, \text { as }}\right)=\frac{2 \pi M}{k}\left(1-\frac{M}{m}\right)\left[\frac{1}{\left(\vec{p}_{1}-\vec{p}_{1}^{\prime}\right)^{2}+M^{2}} \pm \frac{1}{\left(\vec{p}_{1}-\vec{p}_{2}^{\prime}\right)^{2}+M^{2}}\right] .
$$

We see that the symmetrized amplitude gives a pure $S$-wave interaction $\operatorname{Re}\left(A_{\mathrm{S}}\right)=$ $4 \pi(1-M / m) / k M$, while the antisymmetrized contribution is a pure $P$-wave interaction $\operatorname{Re}\left(A_{\mathrm{as}}\right)=4 \pi \vec{p}_{1}^{2} \cos \theta(1-M / m) / k M^{3}$, where $\theta$ is the scattering angle. For any $k<0$, or $k>0$ and $M / m<1$, these amplitudes just represent the expected repulsion between the electrons. However, for $M / m>1$, the magnetic attraction parts (second terms) of these amplitudes, which arise from the Pauli dipole interaction of the electrons due to their magnetic moment [4,6], are stronger than the equal charge-charge Coulomb repulsions (first terms), and lead to a short-ranged equal charge attraction between the electrons. This remarkable equal charge attraction implies that for $M / m>1$ an unusual spectrum of $S$-wave and $P$-wave $e^{-} e^{-}$bound states may exist in the spectrum of the quantum field theory (1), and it may even lead to a possible vacuum instability in the relativistic theory [7], whereby the vacuum could become unstable to the production of $e^{+} e^{-}$pairs followed by a separation of phases. It also implies that there may be a fermion chiral condensate, $<\bar{\psi} \psi>\neq 0$, and the corresponding BCS gap equation for the superconducting ground state (of electron Cooper pairs) can be readily solved in the weak binding case [2].

One can now obtain the potential for the $e^{-} e^{-}$interaction in configuration space. We consider only the $t$-channel amplitude in (3) (i.e. assume the fermions are distinguishable), include the imaginary Aharanov-Bohm part

$$
\operatorname{Im}\left(A_{\mathrm{s}, \mathrm{as}}\right)=\frac{4 \pi M^{2}}{k m} \frac{\vec{q} \times \vec{p}_{1}}{\vec{q}^{2}\left(\vec{q}^{2}+M^{2}\right)}
$$

of (2), and write (2) as a momentum space first order Born amplitude in the momentum transfer $\vec{q}=\vec{p}_{1}-\vec{p}_{1}^{\prime}$. Notice, however, that since the non-relativistic Hamiltonian is quadratic in momentum, the single-photon-exchange approximation is not gauge invariant 
and one must take into account the two-photon exchange contribution. The two dimensional Fourier transform of this resulting Born amplitude gives the configuration space potential for the $e^{-} e^{-}$interaction, and then, including the centrifugal barrier, the effective potential appearing in the corresponding radial Schrödinger equation is

$$
V_{f f}^{(\ell)}(r)=\frac{2 M}{k}\left(1-\frac{M}{m}\right) K_{0}(M r)+\frac{\ell_{R}(r)^{2}}{m r^{2}} .
$$

In (4), $\ell_{R}(r)=\ell-(1 / k)\left[1-M r K_{1}(M r)\right]$ is the radially dependent Aharanov-Bohm renormalization of the usual integer-valued angular momentum quantum number $\ell$ of the $e^{-} e^{-}$pair, $K_{n}(x)$ denotes the irregular modified Bessel function of order $n$ [8], and we assume that $M>m$ in (4). The modified Bessel functions have the asymptotic behaviours $K_{n}(x) \rightarrow 0$ for $x \rightarrow \infty$ and $K_{0}(x) \sim-\log x, K_{1}(x) \sim 1 / x$ for $x \rightarrow 0$. Notice that the term of order $1 / k^{2}$ in the potential (4), which arises from the two-photon exchange diagrams [6], was not taken into account in Refs. [5,9], which lead the authors of those papers to the incorrect conclusion that the effective centrifugal barrier could be attractive. They therefore find $e^{-} e^{-}$bound states for $\beta \ll 1$, contradicting the corresponding $\mathrm{QED}_{3}$ result, which should be correspondent in this regime. Moreover, the bound states found in Refs. [5,9] existed for small $k$, where perturbation theory breaks down and higher order contributions to the scattering amplitude are equally important (so that one cannot neglect terms of order $1 / k^{2}$ as compared to terms of order $\left.1 / k\right)^{1}$.

We employ a standard semi-classical analysis to the potential (4) [10], which yields exact results for the harmonic oscillator and hydrogen atom Hamiltonians. In the WKB approximation, the Bohr-Sommerfeld quantization condition for the radial Schrödinger action integral reads

$$
\int_{r_{1}}^{r_{2}} d r \sqrt{m\left(E_{n, \ell}-V_{f f}^{(\ell)}(r)\right)}=\left(n+\frac{1}{2}\right) \pi \quad ; \quad n=0,1,2, \ldots,
$$

where $r_{1}$ and $r_{2}$ are the classical turning points defined by the zeroes of the function $r^{2}\left(E_{n, \ell}-V_{f f}^{(\ell)}(r)\right), r \geq 0$, and $E_{n, \ell}$ is the mass of the bound state with the quantum numbers $n, \ell$. Introducing the dimensionless parameters $x=M r$ and $\varepsilon_{n, \ell}=E_{n, \ell} / M$, from (4) the quantization law (5) can be written as

$$
\beta^{-1 / 2} \int_{x_{1}}^{x_{2}} d x\left[\varepsilon_{n, \ell}-\frac{2}{k}(1-\beta) K_{0}(x)-\frac{\beta}{x^{2}}\left(\ell-\frac{1}{k}\left(1-x K_{1}(x)\right)\right)^{2}\right]^{1 / 2}=\left(n+\frac{1}{2}\right) \pi .
$$

1

We would also like to stress that the original derivation of the equal charge attraction which was done in Ref. [2] was both quantum mechanical and field theoretical (and the field theoretical derivation was repeated in Refs. $[5,9]$ ), contrary to the claim that the field theoretical derivation suggested in those papers is completely different from the results of Ref. [2]. 
We have solved the equation (6) numerically for $\ell=0,1$ and $n=0,1$ using the standard method of successive approximations. We restricted the parameter $\beta$ to the region $1<\beta \leq 15$, and it was found that bound states (i.e. solutions $\varepsilon_{n, \ell}$ to equation (6)) existed only for $0<k \leq 1.6$. In fact, only in the case of the lowest lying $S$-wave state $(n=\ell=0)$ did $k$ cover the full range $0-1.6$ (in the other cases bound states ceased to exist typically at about $k=0.6$ ). The bound state energies $\varepsilon_{n, \ell}$ are all large for large $\beta$ and small $k$, and as one moves away from this region the values tail off smoothly. Notice, however, that the shift in the centrifugal barrier introduces a large hump in the potential, allowing for metastable bound states. In fact, all of the excited $e^{-} e^{-}$states $(n \geq 1)$ were found to be metastable, and moreover existent for only a small range of $k$-values, this parameter range diminishing for smaller values of $\beta$ (this also occured for $n=0$ at $\beta \sim 1$ where, upon examining (4), bound states are not expected). These numerical results also suggest a parameter dependence of the bound state energies as $\varepsilon_{n, \ell} \sim \beta \mathrm{e}^{-k}$.

It is easy to show that in the perturbative regime $(1 / k \ll 1)$ and in the non-relativistic approximation $(r \gg 1 / m>1 / M)$ the potential (4) is purely repulsive and no bound $e^{-} e^{-}$ states can form. For $1 / k \gtrsim 1$ the perturbative calculations are not reliable, and we do not have an expression for the potential of the interaction between two fermions in this case. But one can see that if one considers the perturbative potential (4) for smaller and smaller $k$, it becomes more and more attractive. Our numerical studies above of this potential indicate that it can possess $S$ - and $P$-wave bound states for $k \lesssim 1$.

Notice also that as $M$ becomes very large (the so-called "anyon limit") the short-ranged potential in (4) becomes an attractive delta-function potential $-(4 \pi / k)(1 / m) \delta^{(2)}(\vec{r})$, and the barrier shift is exactly $\ell \rightarrow \ell-\frac{1}{k}$. Although this delta-function potential admits an $S$-wave bound state $^{2}$, there are no bound states in this limit because the renormalized centrifugal barrier is never absent. This potential just represents the Pauli interaction of the fermionic magnetic moment with the flux tubes attached to the charged particles which transmute them into anyons, and its only effect is to give the electrons fractional spin and statistics $[4,6,12]$. So, we may conclude that as long as we can trust perturbation theory, $e^{-} e^{-}$bound states do not exist in the theory (1). We conjecture that they will form for $M>m$, provided $k$ is sufficiently small, $k \lesssim 1$, and the photon is not too heavy ${ }^{3}$.

For a discussion of the delta-function potential in two spatial dimensions, see Ref. [11].

3 Actually, it was shown in Refs. [4,6] that one-loop radiative corrections give the region of dominant magnetic attraction between two equally charged fermions as $k<-\frac{7}{3}$. We neglect these finite renormalizations of all bare parameters appearing in (1). 
In a similar fashion one can find the potential for the $e^{+} e^{-}$interaction. The tree-level Feynman amplitude in the perturbation expansion for $e^{+} e^{-}$scattering is given by the sum of the corresponding $t$ - and $s$-channel (annihilation) diagrams:

$$
\begin{aligned}
V_{f \bar{f}}=i\left[\left(\bar{u}\left(p_{1}^{\prime}\right) \gamma^{\mu} u\left(p_{1}\right)\right)\left(\bar{v}\left(p_{2}\right) \gamma^{\nu} v\left(p_{2}^{\prime}\right)\right) G_{\mu \nu}\left(p_{1}-p_{1}^{\prime}\right)\right. & \\
& \left.-\left(\bar{v}\left(p_{2}\right) \gamma^{\mu} u\left(p_{1}\right)\right)\left(\bar{u}\left(p_{1}^{\prime}\right) \gamma^{\nu} v\left(p_{2}^{\prime}\right)\right) G_{\mu \nu}\left(p_{1}+p_{2}\right)\right]
\end{aligned}
$$

where

$$
v(p)=\frac{1}{\sqrt{2 m(E+m)}}\left(\begin{array}{c}
-i\left(p_{1}-i p_{2}\right) \\
E+m
\end{array}\right)
$$

are the on-shell negative energy Dirac spinors with $\bar{v}(p) v(p)=1$ [6]. Again the imaginary part of (8) represents the Aharanov-Bohm flux tube interaction between the charged particles, and in the center of mass frame and in the non-relativistic limit, its real part reads

$$
\operatorname{Re}\left(V_{f \bar{f}}(\vec{q})\right)=-\frac{4 \pi M}{k}\left[\left(1-\frac{M}{m}\right) \frac{1}{\vec{q}^{2}+M^{2}}+\frac{1}{m(2 m-M)}\right] .
$$

We see that for any $k<0$, or $k>0$ and $M / m<1$, the amplitude (9) represents an $e^{+} e^{-}$attraction, and the expected $S$ - and $P$-wave $e^{+} e^{-}$bound states may appear. For $1<M / m<2$, the $\vec{q}$-independent ( $s$-channel) term in (9) is still attractive and dominates over the other $t$-channel term. This term corresponds to a delta-function potential in configuration space, which is known to have $S$-wave bound states even for weak coupling [11]. Therefore $e^{+} e^{-}$bound states can exist in either $S$-wave or $P$-wave, provided that either $k<0$ or the photon is stable. However, if the photon is unstable, then the $s$-channel and magnetic interaction terms in (9) dominate the Coulomb charge attraction between the electron and positron. Then the $e^{+} e^{-}$pair no longer attract, and a stable $e^{+} e^{-}$bound state is not possible, as one would expect for unstable photons.

As a function of the topological mass $M$, the attraction represented by (9) is strongest just below the two-fermion threshold $M \sim 2 m+0^{-}$, and near zero photon mass $M \sim 0^{ \pm}$ where the topologically massive gauge theory (1) degenerates into ordinary $\mathrm{QED}_{3}$, precisely where one would expect $e^{+} e^{-}$bound states to exist. The strongest repulsion between the electron and positron occurs just above the two-fermion threshold $M \sim 2 m+0^{+}$, where the photon becomes unstable and the structure of the quantum field theory (1) begins to deviate enormously from that of usual $\mathrm{QED}_{3}$. It is therefore expected that the spectrum of stable $e^{+} e^{-}$bound states will be concentrated in the regions $M \sim 2 m+0^{-}$and $M \sim 0^{ \pm}$. Notice also that in the anyon limit the short-ranged interaction (9) becomes the (now 
repulsive) delta-function interaction discussed before, and all that remains of the $e^{+} e^{-}$ interaction is the pair's anomalous statistics and the usual fractional shift in the canonical angular momentum.

The effective configuration space interaction potential appearing in the radial Schrödinger equation for the $e^{+} e^{-}$system corresponding to the Born amplitude (8) is

$$
V_{f \bar{f}}^{(\ell)}(\vec{r})=-\frac{2 M}{k}\left[\left(1-\frac{M}{m}\right) K_{0}(M r)+\frac{2 \pi}{m(2 m-M)} \delta^{(2)}(\vec{r})\right]+\frac{\ell_{R}(r)^{2}}{m r^{2}}
$$

where we take $0<M / m<2$ in (10). Notice that in this regime and for $1 / k \ll 1$ the barrier shift is small and it vanishes for $M \rightarrow 0$. Now the delta-function in (10) acts only on $S$ wave states as a constant shift in the corresponding $S$-wave energy eigenvalues. Therefore this term, which represents the $s$-channel annihilation of the electron and positron, can be neglected in the ensuing bound state analysis, remembering to just shift all $S$-wave energy levels by $-\left(M^{2} / m\right)\left(\mathrm{e}^{k(2 m-M) / 2 M}-1\right)^{-1}$. We are therefore left with the same Bohr-Sommerfeld equation (6) to solve, except that now $\beta<1$ and of course the sign of the Bessel function $K_{0}(x)$ in (6) changes. A similar numerical analysis now as that of (6) supports the idea of the existence of $S$ - and $P$-wave $e^{+} e^{-}$bound states in this regime of the theory.

A somewhat more analytic analysis of equation (6) for the $e^{+} e^{-}$system can be performed near the zero photon mass threshold $M \rightarrow 0$ (which also gives the corresponding result in ordinary $\mathrm{QED}_{3}$ where $M$ then plays the role of an infrared cutoff). For $M \ll m$, the short-ranged potential in (10) is nothing but the attractive logarithmic Coulomb potential in two spatial dimensions, which is known to have a discrete eigenvalue spectrum bounded from below with eigenvalues of finite multiplicity [13]. In this case the equation (6) can be written to leading order in $\beta$ as

$$
\int_{y_{1}}^{y_{2}} d y\left[-\ell^{2}-\frac{2}{k \beta} \mathrm{e}^{k \varepsilon_{n, \ell}}(1-\beta) y \mathrm{e}^{2 y}\right]^{1 / 2}+\mathrm{O}\left(\beta^{1 / 2}\right)=\left(n+\frac{1}{2}\right) \pi,
$$

where $y=\log x-k \varepsilon_{n, \ell} / 2(1-\beta)$ and $y_{1}$ and $y_{2}$ are the two zeroes of the function $\ell^{2}+$ $(2 / k \beta)(1-\beta) \mathrm{e}^{k \varepsilon_{n, \ell}} y \mathrm{e}^{2 y}, y \in \mathbf{R}^{1}$, so that $y_{1} \sim-\infty$ and $y_{2} \sim 0$ for $\beta \rightarrow 0$. This expression can be integrated with relative ease and evaluated for $\ell=0,1$ to yield approximate expressions for the masses of the $S$ - and $P$-wave $e^{+} e^{-}$bound states:

$$
\begin{aligned}
& E_{n, \ell=0}^{(f \bar{f})} \simeq \frac{2 M}{k} \log \left[\sqrt{\frac{\pi k M}{m}}\left(n+\frac{1}{2}\right)\right] \\
& E_{n, \ell=1}^{(f \bar{f})} \simeq \frac{2 M}{k} \log \left[\left(\frac{3 \sqrt{2 \mathrm{e}}}{\sqrt{\mathrm{e}}+3 \sqrt{2}}\right) \sqrt{\frac{M k}{2 m}}\left(n+\frac{1}{2}\right) \pi\right] .
\end{aligned}
$$


Then (11) also shows that the number of $S$ - and $P$-wave $e^{+} e^{-}$bound states are $N_{\ell=0} \sim$ $\sqrt{m / \pi k M}$ and $N_{\ell=1} \sim \sqrt{2 m / k M}$ respectively, which are both quite large.

Thus for $M / m<2$ the $e^{+} e^{-}$spectrum of states is qualitatively similar to that expected in ordinary $\mathrm{QED}_{3}$, with quite a large number of $e^{+} e^{-}$bound states appearing. Although the existence of $e^{+} e^{-}$bound states in topologically massive quantum electrodynamics for stable photons may not seem perplexing, the above results being standard in the analysis of the two dimensional Coulomb potential, the existence of another stable and neutral vector particle other than the stable photon can lead to interesting consequences for the structure of the quantum field theory (1). In particular, although it is known from the Coleman-Hill theorem [14] that, when the matter fields have a finite mass gap [15], the statistics parameter does not renormalize beyond one-loop order in perturbation theory with respect to the topologically massive photon, it could be further renormalized by the $P$-wave $e^{+} e^{-}$bound state (for one knows that vector particles in general do contribute to this renormalization $[16])$.

The final elementary interaction one could consider for the quantum field theory (1) is the Compton scattering of an electron and a photon. The photon, being a massive vector excitation in this theory, allows us to treat this two-body interaction using standard methods, as above. The total Compton $S$-matrix element in the tree approximation is the sum of the $s$ - and $u$-channel diagrams for the $e^{-} \gamma$ interaction:

$$
\begin{aligned}
& V_{f \gamma}=-i\left[\left(\bar{u}\left(p_{1}^{\prime}\right) \gamma^{\nu} S\left(p_{1}+p_{2}\right) \gamma^{\mu} u\left(p_{1}\right)\right) e_{\mu}\left(p_{2}\right) e_{\nu}^{*}\left(p_{2}^{\prime}\right)\right. \\
& \left.\quad+\left(\bar{u}\left(p_{1}^{\prime}\right) \gamma^{\nu} S\left(p_{1}-p_{2}^{\prime}\right) \gamma^{\mu} u\left(p_{1}\right)\right) e_{\nu}\left(p_{2}\right) e_{\mu}^{*}\left(p_{2}^{\prime}\right)\right]
\end{aligned}
$$

where $S(p)=i\left(p_{\mu} \gamma^{\mu}-m\right)^{-1}$ is the free fermion propagator, and

$$
e(p)=\frac{1}{\sqrt{2} M|\vec{p}|}\left(\begin{array}{c}
\vec{p}^{2} \\
E p_{1}-i M p_{2} \\
E p_{2}+i M p_{1}
\end{array}\right)
$$

are the on-shell polarization vectors for the photons in the transverse Landau gauge with $p \cdot e(p)=0=e(p) \cdot e(p)$ and $e(p) \cdot e^{*}(p)=-1$ [17]. In the center of mass frame, it is found that only the $u$-channel (second term) part of (12) contributes in the non-relativistic limit:

$$
V_{f \gamma}(\vec{q})=-\frac{2 M}{\vec{q}^{2}+\mu^{2}} \cos \theta+\frac{4 i M \operatorname{sign}(M)}{\vec{q}^{2}\left(\vec{q}^{2}+\mu^{2}\right)} \vec{q} \times \vec{p}_{1}(1-\cos \theta),
$$

where we have introduced the mass parameter $\mu=\sqrt{M(M-2 m)}$. 
In the extreme non-relativistic limit $\vec{q}^{2} \rightarrow 0$ this potential becomes

$$
\left.V_{f \gamma}\right|_{\vec{q}^{2} \rightarrow 0}=\frac{2}{2 m-M} \mathrm{e}^{i \operatorname{sign}(M) \theta}
$$

Thus, for stable photons the $e^{-} \gamma$ pair repel each other, no bound state can form, and the entire spectrum of the quantum field theory (1) is identical in nature to that in ordinary $(2+1)$ (or $(3+1))$ dimensional quantum electrodynamics. But if the photon is unstable, the $e^{-} \gamma$ pair attract each other in $P$-wave, and an $e^{-} \gamma$ bound state may form. It is in this unstable region $M / m>2$ that the spectrum of the theory (1) becomes somewhat exotic and differs enormously from what one would usually expect.

The interaction amplitude (14) describes an extremely strong attraction between the electron and photon for values of the topological mass just above the two fermion threshold, $M \sim 2 m+0^{+}$. Just below this threshold, the electron and photon repel each other very strongly (notice that this is exactly opposite to the case of the $e^{+} e^{-}$interaction and is similar to the $e^{-} e^{-}$interaction). For a binding energy $E=-E_{b}^{(f \gamma)}<0$, it is easy to see that the condition for the formation of a stable $e^{-} \gamma$ bound state is just $E_{b}^{(f \gamma)}>M-2 m$, and thus stable $e^{-} \gamma$ bound states could form even though the photon itself is not a stable particle in this regime of the theory. Also notice that in the anyon limit the interaction amplitude (14) vanishes, and the stability condition $E_{b}^{(f \gamma)}>M-2 m$ is violated. Therefore no stable $e^{-} \gamma$ bound state can form in the anyon limit.

The Fourier transform of (13) gives the non-central configuration space effective interaction potential

$$
\begin{gathered}
V_{f \gamma}^{(\ell)}(r, \phi)=-\frac{2 M}{\pi \mu^{2} r^{2}}\left\{\ell\left(1-\mu r K_{1}(\mu r)\right)+\cos 2 \phi\left[\left(\frac{2 \ell}{\mu r}\left(\mu^{2} r^{2}-2\right)-\mu r\right) K_{1}(\mu r)\right.\right. \\
\left.\left.-\left(\mu^{2} r^{2}+2 \ell\right) K_{0}(\mu r)+1\right]\right\}+\frac{\ell^{2}}{2 m^{*} r^{2}}
\end{gathered}
$$

in the $e^{-} \gamma$ radial Schrödinger equation, where $(r, \phi)$ are the polar coordinates of the relative $e^{-} \gamma$ position vector $\vec{r}$ and $m^{*}=m M /(m+M)$ is the reduced mass of the $e^{-} \gamma$ system. We assume that $M>2 m$ in (15).

Notice that the imaginary part of (13) is similar to (but is not exactly) the AharanovBohm interaction amplitude. Notice also that in the anyon limit $M \gg 2 m, \mu \rightarrow \infty$, and the expression (15) becomes $\left.V_{f \gamma}^{(\ell)}(r, \phi)\right|_{\mu \rightarrow \infty}=-\left(2 M / \pi \mu^{2} r^{2}\right)(\ell+\cos 2 \phi)+\ell^{2} / 2 m r^{2}$. This represents a shift in the orbital angular momentum of the $e^{-} \gamma$ composite system of 
$\ell \rightarrow \ell-\frac{2 m}{\pi M}$. As $M \gg 2 m$ this shift is small and negligible except for the $S$-wave channel $(\ell=0)$, just as for the $e^{-} e^{-}$system when $k$ is large. Therefore the $e^{-} \gamma$ system has an unusual spin-statistics transmutation in the anyon limit which is small, but nevertheless a non-trivial adiabatical rotation through $2 \pi$ of the $e^{-} \gamma$ system as a function its radius $r$ exists and can be computed via (14).

The other limiting case is $M \sim 2 m+0^{+}\left(\mu \rightarrow 0^{+}\right)$. In this limit

$$
\left.V_{f \gamma}^{(\ell)}(r, \phi)\right|_{\mu \rightarrow 0^{+}}=-\frac{2 M}{\pi \mu^{2} r^{2}} \cos 2 \phi\left[1+2 \ell\left(\log \mu r-\frac{2}{(\mu r)^{2}}\right)\right]+\frac{3 \ell^{2}}{4 m r^{2}} .
$$

This potential (without the centrifugal barrier) is completely angle dependent, and it is here that the most and strongest $e^{-} \gamma$ bound states are expected to be concentrated.

In the general case (15), as well as in the limiting case (16), an analysis of the corresponding Schrödinger equation as for the $e^{-} e^{-}$and $e^{+} e^{-}$systems appears to be quite difficult due to the non-trivial angular dependence of $V_{f \gamma}^{(\ell)}(r, \phi)$, which has the form of a dipole or quadrupole interaction. Moreover, it is not clear how this angular dependence affects the binding energies. Nonetheless, a contour plot shows that the potential (15) has very deep wells in certain regions of the polar $(r, \phi)$ plane, and it is thus not unreasonable to speculate that at least for a certain set of parameters, the potential (15) has stable bound states. It is also interesting, from examining the small $r$ divergent behaviour of the Bessel functions in (15), that the $e^{-} \gamma$ system can undergo a falling into the center. Moreover, if $e^{-} \gamma$ and $e^{+} \gamma$ bound states exist, then the relativistic vacuum attracts electrons and positrons to itself, and an ensuing vacuum instability can occur, much in the same way as for the unusual $e^{-} e^{-}$attraction. Whether or not the charged composite $e^{-} \gamma$ bound state can keep on attracting electrons or positrons to itself is another question. This provides evidence that the analysis of the quantum field theory (1) must be regarded as a true many-body quantum field theory problem (not just a quantum mechanical one), and the possibility of $e^{-} \gamma$ and $e^{+} \gamma$ bound states in this theory represents a new novel field theoretical phenomenon.

We see therefore that the spectrum of topologically massive spinor electrodynamics separates into different phases, with critical phase transition points at $M / m=1$ and $M / m=2$ separating the expected spectrum from the exotic one. In the limit of a small topological mass, the spectrum contains only the usual particles expected from ordinary $\mathrm{QED}_{3}$ (or $\mathrm{QED}_{4}$ ), while at these critical phase transition points unusual electron and photon composite particles can emerge in the spectrum. In the anyon limit, the only 
effect of these particle interactions is to give the composite particle systems exotic spinstatistics transmutations, as expected from the effective quantum field theories of anyonic systems [12], and the only stable particle excitations which survive are those induced by the fundamental fermion field $\psi$.

We close by noting that in the scalar version of the quantum field theory (1), the exotic spectral structure discussed above is not possible. Consider the Lagrangian (1), except now with gauge coupling to scalar matter fields $\varphi$ :

$$
\mathcal{L}_{S}=-\frac{1}{4 e^{2}} F_{\mu \nu} F^{\mu \nu}+\frac{k}{8 \pi} \epsilon^{\mu \nu \lambda} A_{\mu} \partial_{\nu} A_{\lambda}+\left(\mathcal{D}_{\mu} \varphi\right)^{\dagger}\left(\mathcal{D}^{\mu} \varphi\right)-m_{S}^{2} \varphi^{\dagger} \varphi
$$

where $m_{S}$ is the meson mass. The meson-meson, meson-antimeson, and meson-photon $S$-matrix elements in the tree-approximation are respectively

$$
\begin{aligned}
V_{m m}= & \operatorname{Re}\left[-i\left(p_{1}+p_{1}^{\prime}\right)^{\mu}\left(p_{2}+p_{2}^{\prime}\right)^{\nu} G_{\mu \nu}\left(p_{1}-p_{1}^{\prime}\right)\right]=\frac{16 \pi M}{k} \frac{m_{S}^{2}}{\vec{q}^{2}+M^{2}} \\
V_{m \bar{m}}= & \operatorname{Re}\left[i\left(p_{1}+p_{1}^{\prime}\right)^{\mu}\left(p_{2}+p_{2}^{\prime}\right)^{\nu} G_{\mu \nu}\left(p_{1}-p_{1}^{\prime}\right)+i\left(p_{1}-p_{2}\right)^{\mu}\left(p_{1}^{\prime}-p_{2}^{\prime}\right)^{\nu} G_{\mu \nu}\left(p_{1}+p_{2}\right)\right] \\
= & -\frac{16 \pi M}{k}\left(\frac{m_{S}^{2}}{\vec{q}^{2}+M^{2}}+\frac{\vec{p}_{1} \cdot \vec{p}_{1}^{\prime}}{M^{2}-4 m_{S}^{2}}\right) \\
V_{m \gamma}= & -i\left[\left(2 p_{1}+p_{2}\right)^{\mu}\left(2 p_{1}^{\prime}+p_{2}^{\prime}\right)^{\nu} e_{\mu}\left(p_{2}\right) e_{\nu}^{*}\left(p_{2}^{\prime}\right) D\left(p_{1}+p_{2}\right)\right. \\
& \left.\quad+\left(2 p_{1}-p_{2}^{\prime}\right)^{\mu}\left(2 p_{1}^{\prime}-p_{2}\right)^{\nu} e_{\nu}\left(p_{2}\right) e_{\mu}^{*}\left(p_{2}^{\prime}\right) D\left(p_{1}-p_{2}^{\prime}\right)-2 i g^{\mu \nu} e_{\mu}\left(p_{2}\right) e_{\nu}^{*}\left(p_{2}^{\prime}\right)\right] \\
= & 2 \mathrm{e}^{i \operatorname{sign}(M) \theta}
\end{aligned}
$$

in the center of mass frame and in the non-relativistic limit. Here $D(p)=i\left(p^{2}-m_{S}^{2}+i \epsilon\right)^{-1}$ is the free scalar propagator, and we have taken $\vec{q}^{2} \rightarrow 0$ in the final amplitude $V_{m \gamma}$ of (18).

The meson-meson pair therefore always repel for any values of the parameters, while the meson and antimeson always attract each other. This occurs because spinless particles have no magnetic moment at tree-level, and hence no unusual Chern-Simons magnetic interaction between them, and they therefore only interact through the usual Coulombic forces (unlike spinor particles). The meson-photon pair also always repel each other in $P$-wave, since in the relevant limits the repulsive bare four-point vertex (third term of $V_{m \gamma}$ in (18)) dominates the total meson-photon interaction (whereas in the spinor case the photon can only dress the fermions). Thus topologically massive scalar electrodynamics can not admit exotic bound states, and the spectrum of the scalar quantum field theory (17) contains precisely the same particles as the ordinary three (or four) dimensional scalar Maxwell theory does. 
Acknowledgements: The authors wish to thank M. Bergeron and A. Vainshtein for helpful discussions. The work of M. D., G. S. and R. S. was supported in part by the Natural Sciences and Engineering Research Council of Canada. The work of I. K. was supported by the U. S. National Science Foundation grant \# NSF PHY90-21984 and that of D. E. was supported by a grant from the U. S. Department of Energy. 


\section{References}

[1] J. F. Schonfeld, Nucl. Phys. B185 (1981), 157; S. Deser, R. Jackiw and S. Templeton, Phys. Rev. Lett. 48 (1982), 475; Ann. Phys. (N.Y.) 140 (1982), 372.

[2] I. I. Kogan, JETP Lett. 49 (1989), 225.

[3] A. Groshev and E. R. Poppitz, Phys. Lett. B235 (1990), 336.

[4] I. I. Kogan, Phys. Lett. B262 (1991), 83; J. Stern, Phys. Lett. B265 (1991), 119; I. I. Kogan and G. W. Semenoff, Nucl. Phys. B368 (1992), 718.

[5] H. O. Girotti, M. Gomes and A. J. da Silva, Phys. Lett. B274 (1992), 357.

[6] R. J. Szabo, I. I. Kogan and G. W. Semenoff, Nucl. Phys. B [FS] (1992), in press.

[7] I. I. Kogan and I. V. Polyubin, SSC preprint SSCL-SR-284 (1990) (unpublished).

[8] I. S. Gradshteyn and I. M. Rhyzhik, Table of Integrals, Series, and Products, Academic Press (San Diego) (1980).

[9] H. O. Girotti, M. Gomes, J. L. deLyra, J. R. S. Nascimento and A. J. da Silva, Phys. Rev. Lett. 69 (1992), 2623; "Electron-Electron Bound States in QED 3 ", University of Sao Paulo preprint IFUSP/P-1012 (1992).

[10] A. Messiah, Quantum Mechanics I, John Wiley and Sons (New York) (1976).

[11] K. Huang, Quarks, Leptons, and Gauge Fields, World Scientific (Singapore) (1982).

[12] F. Wilczek and A. Zee, Phys. Rev. Lett. 51 (1983), 2250; G. W. Semenoff, Phys. Rev. Lett. 61 (1988), 517; T. Matsuyama, Phys. Lett. B228 (1989), 99; G. W. Semenoff and P. Sodano, Nucl. Phys. B328 (1989), 753; S. Forte and T. Jolicoeur, Nucl. Phys. B350 (1991), 589.

[13] F. Geszetesy and L. Pittner, J. Phys. A11 (1978), 679.

[14] S. Coleman and B. Hill, Phys. Lett. B159 (1985), 184; Y. Kao and M. Suzuki, Phys. Rev. D31 (1985), 2137; M. Bernstein and T. Lee, Phys. Rev. D32, (1985), 1020.

[15] G. W. Semenoff, P. Sodano and Y.-S. Wu, Phys. Rev. Lett. 62 (1989), 715.

[16] R. Jackiw and S. Templeton, Phys. Rev. D23 (1981), 2291.

[17] I. I. Kogan and A. Morozov, Sov. Phys. JETP 61 (1985), 1. 